\title{
Towards conformant models of automated electric vehicles
}

Ray Lattarulo ; Daniel Heß ; Jose A. Matute ; Joshue Perez

2018, Page(s):1-6

(C) 2018 IEEE. Personal use of this material is permitted. Permission from IEEE must be obtained for all other uses, in any current or future media, including reprinting/republishing this material for advertising or promotional purposes, creating new collective works, for resale or redistribution to servers or lists, or reuse of any copyrighted component of this work in other works. "

https://ieeexplore.ieee.org/document/8519484 


\title{
Towards conformant models of automated electric vehicles
}

\author{
Ray Lattarulo ${ }^{\mathrm{a}, \mathrm{c}}$, Daniel Heß ${ }^{\mathrm{b}}$, Jose A. Matute ${ }^{\mathrm{a}, \mathrm{c}}$, Joshue Perez ${ }^{\mathrm{a}}$ \\ ${ }^{a}$ Industry and Transport Division, Tecnalia Research \& Innovation, San Sebastian, Spain \\ ${ }^{b}$ Deutsches Zentrum für Luft-und. Raumfahrt (DLR), Germany \\ ${ }^{c}$ Automatic Control and System Engineering Department, University of the Basque Country, Bilbao, Spain \\ rayalejandro.lattarulo@tecnalia.com, daniel.hess@dlr.com, joseangel.matute@tecnalia.com, joshue.perez@tecnalia.com
}

\begin{abstract}
Automated driving is one of the major tendencies in last decades, and it is presented as a reliable option to improve comfort during driving, including disable and elder in society and increasing persons safety in roads. This last topic produces the question how is it possible to verify planning and control algorithms for a reliable commercial use of this technology. The question can be answered from two perspective: experimental or formal methods, where the formal one is selected as the most robust between both. Hence, the current work presents a case study verification in automated driving for lane change and double lane change maneuvers, using as basis a trace conformance method presented in [1]. The verification method is performed in Dynacar as a precise multibody simulator tuned for a commercial Renault Twizy vehicle.

Index Terms-Automated vehicles, conformance testing, tracking controllers, verification.
\end{abstract}

\section{INTRODUCTION}

Last decades have seen great approaches and advances in the automotive sector. Some of the most relevant were Advanced Driver Assistance Systems (ADAS), that has been boosted by a great amount of challenges, projects and initiatives in public and private sectors. Years later, these systems evolved to set up automated driving (AD). Some highlight examples in AD were NavLab (Carnegie Mellon University) demonstrations [2] and DARPA Challenges [3]. Improvement in environment perception [4], communication along multiple participants (V2X) [5], different types of planning algorithms [6] and trajectory and speed tracking controllers [7] are some examples resolved at researching level, but there is still a lack of verification and validation of this technology.

Automated vehicles can be considered within the CyberPhysical Systems (CPS) category. The CPS are all those systems where an interaction of embedded systems (automation and control part) and physical process of the plant (system) exists, in such way that all of them are constituted by three basic parts: i) computer and software, ii) a network structure among multiple computers and systems involved in the plant and iii) the physical process part [8]. In general, the union of these parts is a key point of study in CPS, including the specifications, modeling, designing, programming, performance analysis, testing, debugging, verification and validation. The last two points have major relevance in this technology, for the safety critical operation of most of them [9], but they are particularly difficult to be done for simultaneous interactions between analog (physical) and digital (computers) parts [10].

For software and hardware systems, experimental and formal verification are forms of verifying system robustness during design time [11]. One of the major problems of experimental testing is than ambiguities and conflict can appear. Whereas formal verification reduces those problems. The system will be robust enough and reliable if the model is exact in certain degree with respect to the plant [12].

Some authors have studied formal verification in mobile robots. In [13] DRONA (a software toolchain) was used for formal verification of Distributed Mobile Robots (DMR). The authors studied the use case of delivery robots, permitting them to find several errors and bugs that cannot be easily found during testing. The toolbox was designed to support distributed and asynchronous systems.

It is relevant to understand that software testing and conformance is based on a blackbox approach [14]. Therefore [15] studied blackbox conformance under real-time targets. The blackbox component is related to lack of information or knowledge of system under test (SUT) and the conformance is done with knowledge of the input and output traces for partially observable and non-deterministic systems [15].

The current work presents an automated vehicle verification approach based on trace conformance and its basis are in the work done by [1]. In this paper, the trace conformance is mainly to verify the abstract vehicle model used to control the system with robustness. The vehicle used in this paper is a multi-body model tuned with a commercial vehicle (Renault Twizy) parameters. Additionally, the work considers the generation of a feasible trajectory based on parametric Bézier curves and implemented in a modular architecture [16].

The rest of this paper is organized as follows: Section II presents the project framework of the current work, and the verification approach based on trace conformance, considering a modular automated vehicle architecture. Section III gives information related with the multi-body vehicle model and the tuning process based on real vehicle information. Section $\mathrm{V}$ is used to verify that a simple inspection is not enough to verify the system giving basis to the trace conformance. Following, Section VI presents the trace conformance analysis and finally, Section VII concludes and proposes future works in terms of the current approach. 


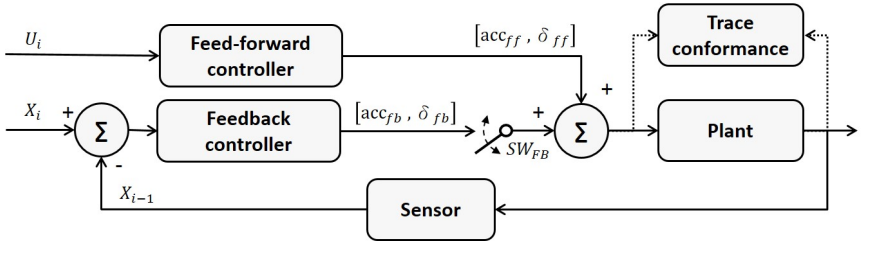

Fig. 1. Complete control scheme (open and close loop)

\section{TRACE CONFORMANCE APPROACH}

The Unifying Control and Verification of Cyber-Physical Systems (UnCoVerCPS) project is an European initiative to reduce the verification time for safety and/or operational critical systems, and unifying control strategies with system verification approaches based on formal methods. Some of the use cases considered for the project are: wind turbines, smart grids, human-robots and automated vehicles [17]. This last one is the goal of the current approach.

Trace conformance is presented as a formal method for system verification. It relies in the trace analysis of a test case. The goal is to conform the system's behavior (model used in lateral and longitudinal controllers) based on maximum disturbances. The offline information of input, output and bounds around traces.

A highly precise multi-body vehicle model (plant) was used, in order to make verifications before its implementation on a real vehicle. The Figure 1 shows the architecture for the trace conformance evaluation. The main goal is to use a simplified model of the vehicle as a feed-forward controller considering the trajectory information, and conforming the control with plant behaviors under certain disturbances (in the future these disturbances will be associated to the sensor errors).

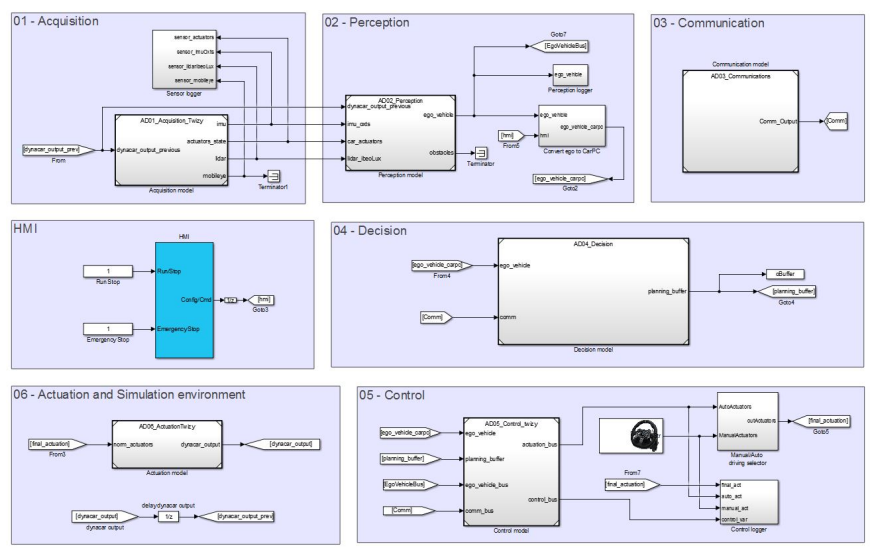

Fig. 2. Automated driving control architecture (software)

The automated vehicle architecture (Fig. 2) uses the description of 6 blocks [6], [16]. The abstraction is defined in several blocks as; acquisition, perception, communication, decision, control and actuation. The trace conformance approach tested in the current work is related with decision (trajectory) and control (feed-forward and feed-back control) modules.
TABLE I

ChaRACTERISTICS RENAULT TWIZY URBAN 80

\begin{tabular}{ll}
\hline Mass & $611.5[\mathrm{~kg}]$ \\
Wheelbase & $1.686[\mathrm{~m}]$ \\
Trackwidth & $1.094[\mathrm{~m}]$ \\
Inertia $\left(I_{x}, I_{y}, I_{z}\right)$ & $243.175,430.166,430.166\left[\mathrm{~kg} . \mathrm{m}^{2}\right]$ \\
Front/Rear wheel radius & $0.265 / 0.281[\mathrm{~m}]$ \\
\hline Motor type & 3-Phase Asynchronous \\
Power & $11[\mathrm{HP}]$ from 220 to $785[\mathrm{rad} / \mathrm{sec}]$ \\
Torque & $57[\mathrm{~N}-\mathrm{m}]$ from 0 to $220[\mathrm{rad} / \mathrm{sec}]$ \\
Transmission & Automatic w/Gear reduction \\
Reduction & $1: 9.23$ \\
\hline Front/Rear brake & Single circuit - Discs \\
\hline
\end{tabular}

The left top side of Figure 1 shows the Acquisition module that is in charge of gathering sensor/simulation raw input data (position, velocity, etc.). In middle top part is Perception, which models the environment and ego-vehicle based on raw data coming from Acquisition. Communication is in the top right side, and it is related with V2X data exchange (infrastructure, pedestrians or other vehicles). Decision is in the right middle part, it is related with the vehicle decisions from macro assignments (going forward, turning left, overtake, etc.) to trajectory and speed planning to be tracked by controllers. Right bottom part is Control, which is separated in lateral (steering) and longitudinal (throttle/brake), in some case they can be considered coupled in one controller. The last block is Actuation in the bottom left side, which comprises low level controllers of steering wheel, throttle and brake systems. Although HMI (middle left side) is not considered as an specific module, it contains important information (databases and configuration parameters).

\section{VEHICLE PLATFORM AND MULTIBODY FORMULATION}

Although the development of this work have been focused on tests in virtual environments, a considerable effort has been done in order to mimic the behavior of a Renault Twizy Urban 80, a real test platform typically used by Tecnalia Automated Driving Group to experiment with ADAS functionalities.

The vehicle is equipped with several devices connected to the chassis that had to be modeled as; anti-roll bars, suspension compliance, shock absorbers, tire characteristics and its road interface. A simplified aerodynamic influence was also considered [18]. The propulsion, braking and steering systems are the most important aspects in the modeling, needing a high level of accuraccy to represent a similar dynamic behavior both in virtual and real platform [19]. Technical parameters of the real platform are shown in Table I.

\section{A. Dynacar as simulation environment}

The virtual platform is developed in Dynacar, a simulation tool for vehicle dynamics based on a multi-body formulation [20]. This formulation is very uselful to obtain different physical parameters, being mainly important the study of passeger's stability and comfort [21]. A module within this tool is dedicated to develop 3D environments as well as perform test visualizations. A comparison between real and simulated platforms is shown in the Figure 3. 


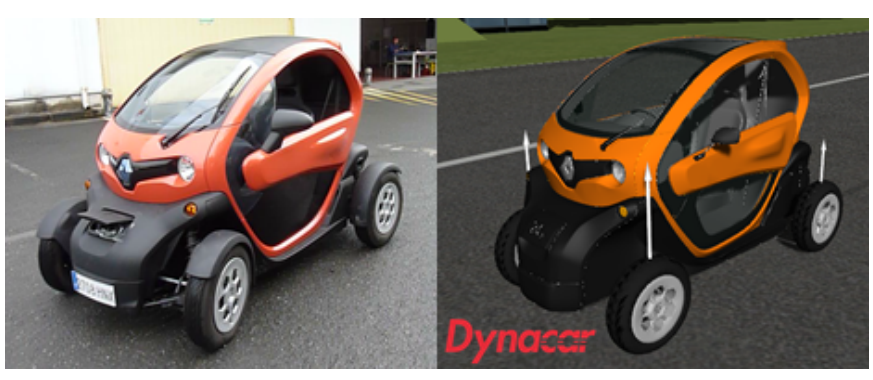

Fig. 3. Real and virtual platforms

TABLE II

Plant PARAMETER IDENTIFICATION

\begin{tabular}{ll}
\hline Inputs in open loop identification & \\
\hline Mass $(\mathrm{M})$ & $582.5[\mathrm{~kg}]$ \\
$J_{m}$ & $0.5150\left[\mathrm{~m}^{2}\right]$ \\
CG location & $0.4502[\mathrm{~m}]$ \\
$C_{f}$ & $0.450[1 / \mathrm{rad}]$ \\
$C_{r}$ & $4.991[1 / \mathrm{rad}]$ \\
$\delta_{\text {ratio }}$ & 15 \\
\hline Plant parameter identification & \\
\hline$I_{z} / \mathrm{M}$ & $0.515\left[\mathrm{~m}^{2}\right]$ \\
b/L & $0.450[-]$ \\
$C_{f}$ & 4.991 \\
$C_{r}$ & 7.512 \\
\end{tabular}

Relative coordinates are used to model the vehicle, where mass matrix and force vector are recursively obtained [22] in order to obtain equations of motion. Each suspension is considered as a macro-joint substituting the suspension links by look-up tables, leading to a tree-like kinematic structure [23]. The forces due to the spring-damper elements have been introduced through the motion-ratio approach [24]. Pacejka semi-empirical approach has been implemented [25], where the tire is characterized by a list of coefficients which can be obtained from experimental tests.

\section{B. Model for feed-forward controller}

A bicycle model has been implemented in order to be used as a feed-forward controller. The model's parameters have been identified with an open-loop model validation, using a lane change and a double lane change manouvers [26] as use cases and comparing the model with multi-body formulation. The steering wheel angle and longitudinal acceleration has been obtained from moving tests of the real platform. The validation was made comparing the states of the bicycle model with experimental data.

\section{DECISION AND CONTROL DEFINITION}

The current section explains all the information related with trajectory generation and the controller used. The scenarios used during trace conformance verification were single and double lane change (maneuvers used normally during emergency situations).

Trajectories will be generated using Bézier curves. They have certain properties interesting for the purposes of current work (further explained in [27]) as: i) the typical "s-shape" of lane changes can be easily achieved using Bézier control points symmetrically located and aligned in the current lane and the next one, ii) the generated curves will lye in the convex hull form by control points (partly knowing where the trajectory is) and iii) they have geometrical and curvature continuity.

In the current work, the trajectory will be named as $U_{i}$ :

$$
U_{i}=\left[x_{i}, y_{i}, \Psi, v_{x}, v_{y}, k, a_{x}\right]^{T}
$$

where $x_{i}$ and $y_{i}$ are coordinates of i-point in trajectory (based on UTM coordinate system), $\Psi$ is the yaw angle, $v_{x}$ and $v_{y}$ are longitudinal and lateral velocities respectively, $k$ is curvature and $a_{x}$ is the longitudinal acceleration.

The tracking control scheme used was a feed-back plus feed-forward for trace conformance. The Figure 1 depicts the block diagram associated. The "Plant" will be the multibody vehicle representation. $U_{i}$ is the trajectory, $X_{i}$ are the plant states at i-sample time, $a c c_{f b}$ and $a c c_{f f}$ are acceleration control signals, and $\delta_{f b}$ and $\delta_{f f}$ are the steering wheel angles for feed-back and feedforward loops respectively.

The feedforward control has a dynamic bicycle representation that can be very precise at low and moderate speeds [28], [29]. In Figure 4 is represented this model with the trajectory. The frontal and rear tire slip angles $\alpha_{f, r}$ are given by:

$$
\begin{aligned}
& \alpha_{f}=\delta_{f}-\frac{v_{y}+\omega a}{v_{x}} \\
& \alpha_{r}=\frac{b \omega}{v_{x}}-\frac{v_{y}}{v_{x}}
\end{aligned}
$$

where $a$ and $b$ are distances from the center of gravity $C O G$ to the front and rear wheel respectively (Fig. 4), $v_{x, y}$ are the longitudinal and lateral velocities, and $\delta_{f}$ is the steering wheel angle. The resulting force over the tire is given by:

$$
C_{f, r}=c_{f, r} \frac{d_{a, b}}{L} M g \rightarrow F_{y_{f, r}}=C_{f, r} \alpha_{f, r}
$$

where $C_{f, r}$ is the cornering stiffness of the frontal and rear tire and $c_{f, r}$ are the normalized values of the cornering.

After, the sum of lateral forces is obtained:

$$
\frac{F_{y t}}{M}=\frac{F_{y f}}{M}+\frac{F_{y r}}{M} \rightarrow \frac{F_{y f}}{M}=\frac{F_{y t}}{M}-\frac{F_{y r}}{M}
$$

Knowing the trajectory values of speed reference $v_{s}$, curvature $k$ and acceleration $a_{x}$ (given in the vector $U_{i}$ ), lateral and longitudinal accelerations contribution can be generated from reference and angle error $e_{\alpha}$ between vehicle and trajectory:

$$
\begin{aligned}
& a_{x t}=a_{x} \\
& a_{y t}=v_{s}^{2} k \cos \left(e_{\alpha}\right)+a_{x} \sin \left(e_{\alpha}\right)
\end{aligned}
$$

Combining Eq. 4 and 5:

$$
c_{f} \frac{a}{L} g\left(\delta_{f f}-\frac{v_{y}+\omega a}{v_{x}}\right)=a_{y t}-\frac{C_{r} \alpha_{r}}{M}
$$

from this equation is obtained the feed-forward contribution for steering wheel angle, using the bicycle model and trajectory information. In case of the longitudinal feed-forward 


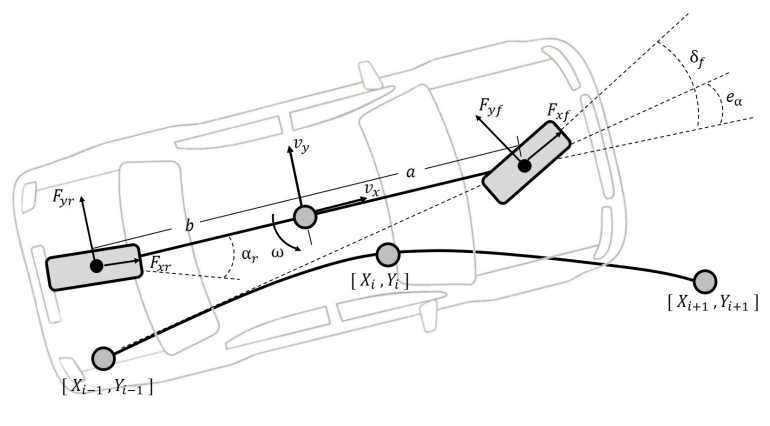

Fig. 4. Bicycle feed-forward model

contribution is used directly, the longitudinal acceleration reference is shown in Equation 5. In case of the feed-back loop, simple linear controllers with lateral and angular corrections can be described in the steering wheel signal. In the case of longitudinal control, a simple correction of position and distance is performed.

\section{OPEN AND CLOSED LOOP PERFORMANCE INSPECTION}

Emergency maneuvers are necessary risky situations. Hence, decision and control in automated driving must fit high standards on these types of maneuvers, and how verify these requirements is still an open question. Therefore, the current section will present maneuvers considered as part of most emergencies while driving, as single and double lane change.

Fig. 5 shows the trajectory (top part) followed by the vehicle in open loop (Fig. 1 with opened switch) under a lane change maneuver. The reference trajectory is shown in thin continued line, the open loop control model (bicycle) and vehicle are shown in dash line (thicker and thinner lines respectively). The states variables orientation $\psi$, yaw rate $\omega$, longitudinal speed $v_{x}$ and lateral speed $v_{y}$ can be analyzed from this behavior. A considerable good performance in comparison with the reference could be concluded, however the trajectory following ( $\mathrm{x}-\mathrm{y}$ coordinates), looks deviated with the reference. In such way, the conclusions obtained from results can be ambiguous.

In the other hand, Fig. 6 shows a closed loop behavior of the system performing a double lane change maneuver, obtaining a better performance than open loop case. The ambiguity relies on feed-back loop responses (clearly seen in $\omega$ and $v_{y}$ oscillations around references) where it is not easy to determine if the system is working properly or not under certain performance parameters.

\section{TRACE CONFORMANCE TESTING RESUltS}

The conformance method is based on [1], where a recorded output trace (of the SUT) is compared with the model for feedforward controller under certain disturbances. If traces errors are into specified bounds the model is trace conformed, but if values are out of bounds the model must be improved due was not able to accomplish the requirements.

The Figures 7 and 8 show trace conformance analysis for double lane change in open and closed loop respectively.
Maximum admissible boundaries are summarized in Table III. In closed loop test the same maneuver is verified and bounds used contain all the states.
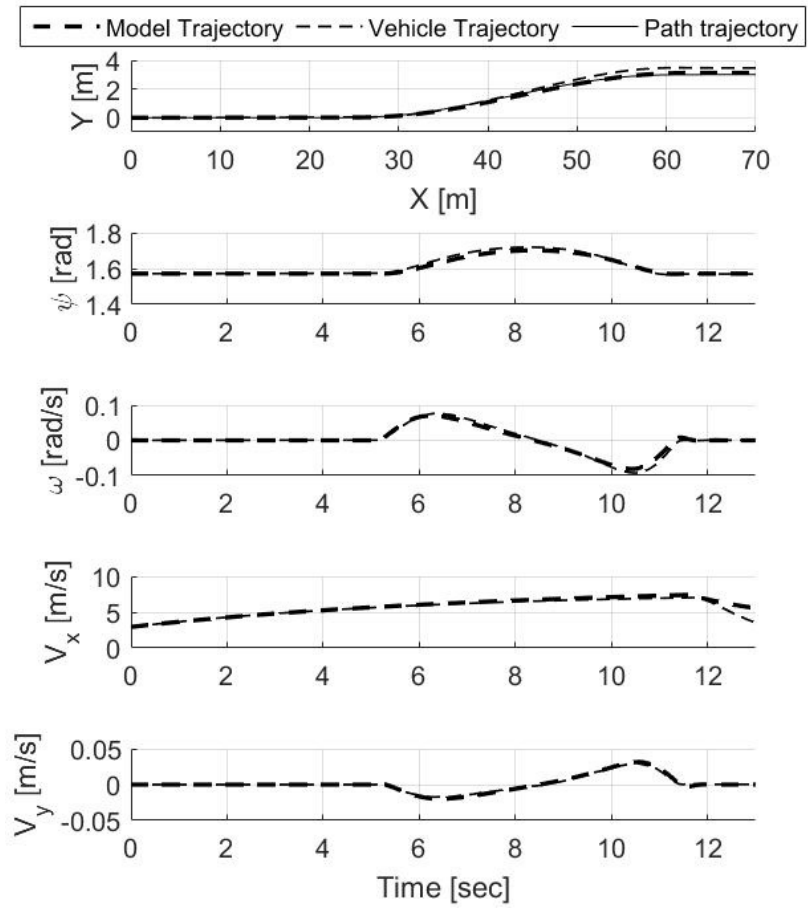

Fig. 5. Trajectory and state variables for open-loop model identification (single lane change)
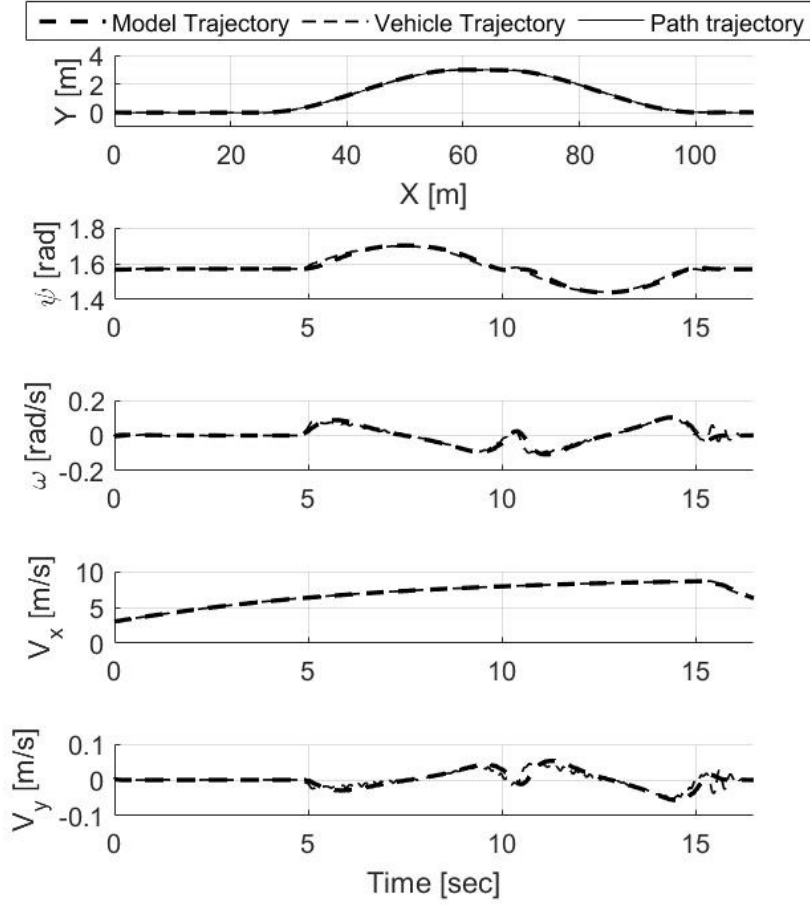

Fig. 6. Trajectory and state variables for closed-loop model identification (double lane change) 

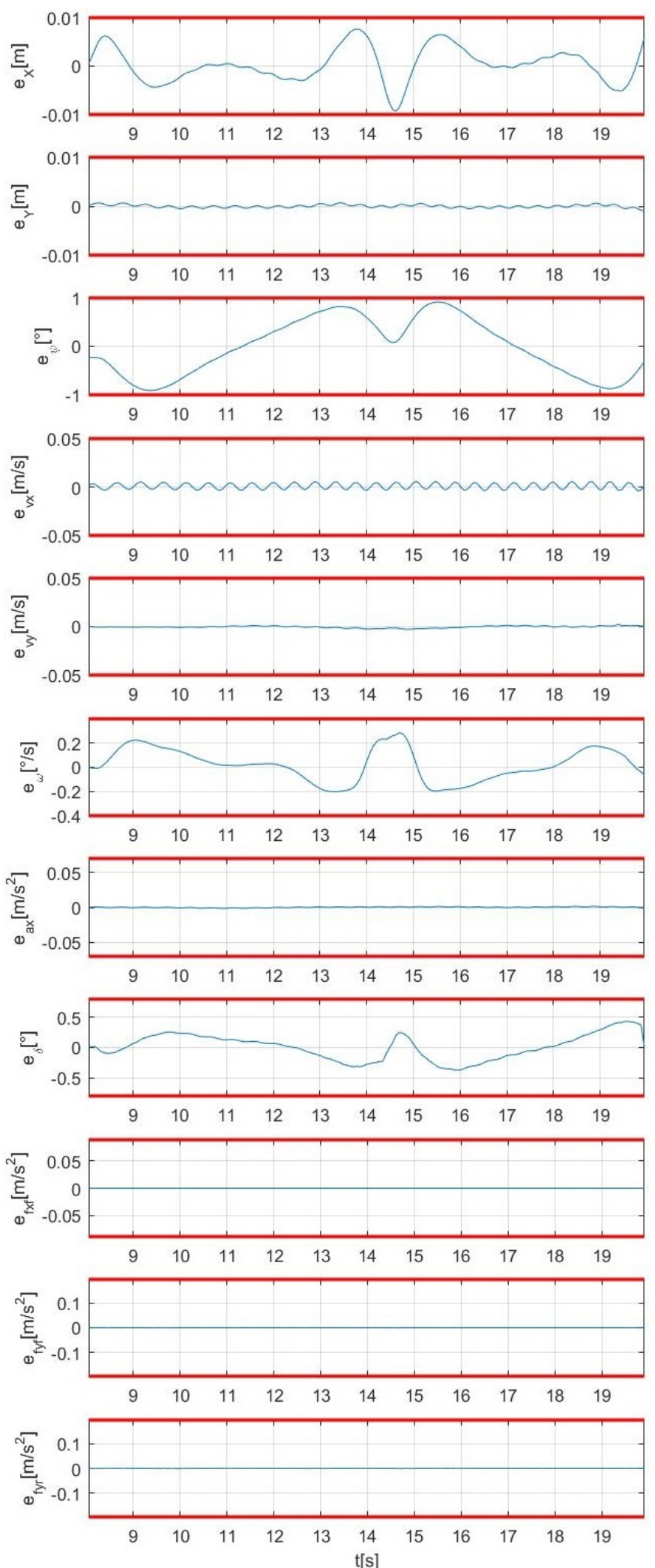

Fig. 7. Open loop conformance testing under double lane change scenario
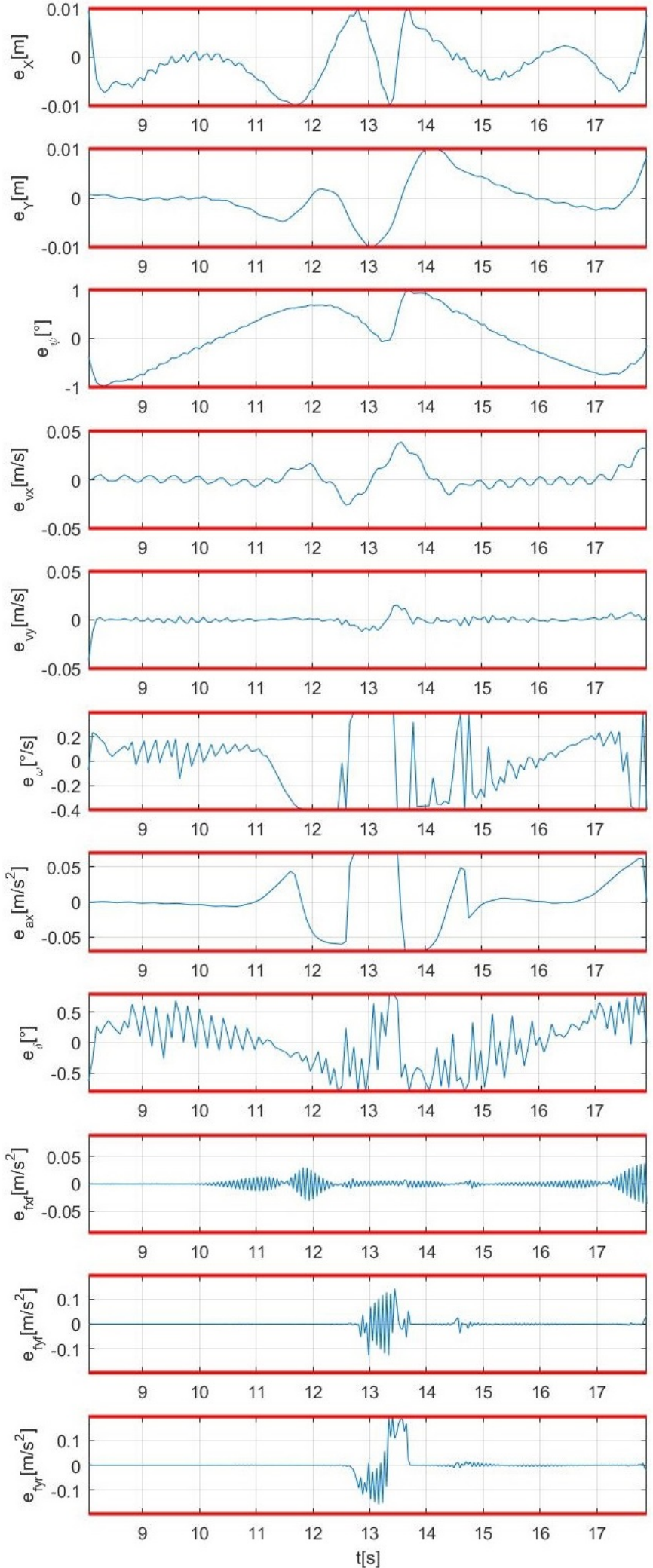

Fig. 8. Closed loop conformance testing under double lane change scenario 
TABLE III

TRACE CONFORMANCE MAXIMUM DISTURBANCES

\begin{tabular}{llllll}
\hline$x[m]$ & 0.01 & $y[m]$ & 0.01 & $\phi\left[^{\circ}\right]$ & 1.00 \\
$v_{x}[\mathrm{~m} / \mathrm{s}]$ & 0.05 & $a_{x}\left[\mathrm{~m} / \mathrm{s}^{2}\right]$ & 0.07 & $\delta\left[^{\circ}\right]$ & 0.7 \\
$f_{x f}\left[\mathrm{~m} / \mathrm{s}^{2}\right]$ & 0.1 & $f_{y f}\left[\mathrm{~m} / \mathrm{s}^{2}\right]$ & 0.2 & $f_{y r}\left[\mathrm{~m} / \mathrm{s}^{2}\right]$ & 0.2 \\
\hline
\end{tabular}

It is relevant to verify the open loop system due to the major contribution will come from this part, and additionally, will be easier to conform the system with minor reactive part contribution in the control system (feed-back loop).

\section{DISCUSSION AND CONCLUSIONS}

Behavior verification in automated driving is one of the most important tasks in upcoming years for this technology. Meanwhile a great amount of effort have been done in the development of; perception algorithm, communication, path planning and tracking controllers, the verification of these systems has not received a proper effort. Experimental methods are possibilities proposed for verification of these kind of systems, however they need a great time for data collection and evaluation. Whereas, the formal method is presented as a better option for verification.

A trace conformance approach was used in the current work in a concept test proof based on a vehicle using Dynacar, showing favorable results applying a feed-forward controller based on a bicycle model for trajectory tracking, showing that if an open loop model is close enough to the reality it contributes with the automated vehicle control, being easier the verification of the system and obtaining less correction behaviors given by the feed-back control.

Future works are related to verify the present approach on a real platform exploring more robust methods, contributing with real platform behavior verifications.

\section{ACKNOWLEDGMENT}

The authors want to thank to the H2020 UnCoVerCPS Project with grant number 643921.

\section{REFERENCES}

[1] B. Schürmann, D. Heß, J. Eilbrecht, O. Stursberg, F. Kö]ster, and M. Althoff, "Ensuring drivability of planned motions using formal methods," IEEE International Conference on Intelligent Transportation Systems (ITSC), October 2017.

[2] G. Dimitrakopoulos, "Chapter 4, advanced driver assistance systems (adas)," Springer Current Technologies in Vehicular Communications, pp. $63-96,2017$.

[3] Ö. Ş. Taş, F. Kuhnt, J. M. Zöllner, and C. Stiller, "Functional system architectures towards fully automated driving," IEEE Intelligent Vehicles Symposium (IV), Jun. 2016.

[4] R. Domínguez, E. Onieva, J. Alonso, J. Villagra, and C. González, "Lidar based perception solution for autonomous vehicles," IEEE 11th International Conference on Intelligent Systems Design and Applications (ISDA), pp. 790 - 795, November 2011.

[5] L. Li, D. Wen, and D. Yao, "A survey of traffic control with vehicular communications," IEEE Transactions on Intelligent Transportation Systems, pp. 425 - 432, 2013.

[6] D. González, J. Pérez, V. Milanés, and F. Nashashibi, "A review of motion planning techniques for automated vehicles," IEEE Transactions on Intelligent Transportation Systems 17(4), pp. 1135 - 1145, 2016.
[7] J. Ni and J. Hu, "Dynamics control of autonomous vehicle at driving limits and experiment on an autonomous formula racing car," ELSEVIER Mechanical Systems and Signal Processing 90, pp. 154 - 174, 2016.

[8] E. A. Lee and S. A. Seshia, "Introduction to embedded systems - a cyber-physical systems approach," LeeSeshia.org, 2011.

[9] S. A. Seshia, S. Hu, W. Li, and Q. Zhu, "Design automation of cyber-physical systems: Challenges, advances, and opportunities," IEEE Transactions on Computer-Aided Design of Integrated Circuits and Systems 36(9), pp. 1421 - 1434, September 2017.

[10] Y. Driouich, M. Parente, and E. Tronci, "Modeling cyber-physical systems for automatic verification," IEEE International Conference on Synthesis, Modeling, Analysis and Simulation Methods and Applications to Circuit Design (SMACD), June 2017.

[11] F. Bessayah, A. Cavalli, and E. Marting, "A formal approach for specification and verification of fault injection process," Proceedings of the 2nd International Conference on Interaction Sciences: Information Technology, Culture and Human, pp. 883 - 890, November 2009.

[12] R. M. Hierons, K. Bogdanov, J. P. Bowen, R. Cleaveland, J. Derrick, J. Dick, M. Gheorghe, M. Harman, K. Kapoor, P. Krause, G. Lüttgen, A. J. H. Simons, S. Vilkomir, M. R. Woodward, and H. Zedan, "Using formal specifications to support testing," ACM Computing Surveys (CSUR) 41(2), pp. 9:1 - 9:76, February 2009.

[13] A. Desai, I. Saha, J. Yang, S. Qadeer, and S. A. Seshia, "Introduction to embedded systems - a cyber-physical systems approach," ACM/IEEE 8th International Conference on Cyber-Physical Systems (ICCPS), pp. 239 - 248, April 2017.

[14] G. V. Bochmann and A. Petrenko, "Protocol testing: Review of methods and relevance for software testing," ACM SIGSOFT international symposium on Software testing and analysis, pp. 109 - 124, August 1994.

[15] M. Krichen and S. Tripakis, "An expressive and implementable formal framework for testing real-time systems," Springer International Conference on Testing of Communicating Systems (TestCom), pp. 209 - 225, 20052005.

[16] R. Lattarulo, J. Pérez, and M. Dendaluce, "A complete framework for developing and testing automated driving controllers," ELSEVIER 20th IFAC World Congress, pp. 258 - 263, 2017.

[17] European Commission CORDIS: Community Research and Development Informantion Service. (2016) Uncovercps report summary. [Online]. Available: https://cordis.europa.eu/result/rcn/190443_en.html

[18] SAE International, "Vehicle dynamics terminology: Sae j670e," Vehicle Dynamics Standards Committee, 2008.

[19] M. Marcano, J. Matute, R. Lattarulo, E. Marti, and J. Perez, "Low speed longitudinal control algorithms for automated vehicles in simulation and real platforms," Complexity, 2018.

[20] J. Cuadrado, D. Vilela, I. Iglesias, A. Martin, and A. Peña, "A multibody model to assess the effect of automotive motor in-wheel configuration on vehicle stability and comfort," ECCOMAS Thematic Conference Multibody Dynamics 2013, pp. 457-458, 2013.

[21] J. Matute, M. Marcano, A. Zubizarreta, and J. Perez, "Longitudinal model predictive control with comfortable speed planner," IEEE International Conference on Autonomous Robot Systems and Competitions (ICARSC2018), 2018.

[22] J. Cuadrado, D. Dopico, M. A. Naya, and M. Gonzalez, "Penalty, semirecursive and hybrid methods for mbs real-time dynamics in the context of structural integrators," Springer Multibody System Dynamics 12(2), pp. 117-132, 2004.

[23] M. Acevedo and J. Celigüeta, "Real-time dynamic simulation of passenger cars," Mechatronics \& Supercomputing Applications in the Transportation Industries (ISATA), pp. 559-566, 1994.

[24] W. Milliken and D. Milliken, "Race car vehicle dynamics," SAE International, 1994.

[25] H. B. Pacejka, Butterworth-Heinemann: Tyre and Vehicle Dynamics 2ed, pp. v - vii, 2006

[26] ISO, "Road vehicles - lateral transient response test methods - openloop test methods," ISO 7401:2011, p. 22, 2011.

[27] R. Lattarulo, M. Marcano, and J. Pérez, "Overtaking maneuver for automated driving using virtual environments," Springer International Conference on Computer Aided Systems Theory, pp. 446 - 453, 2018.

[28] H. Ren, T. Shim, J. Ryu, and S. Chen, "Development of effective bicycle model for wide ranges of vehicle operations," SAE World Congress \& Exhibition, pp. 1 - 9, 2014.

[29] R. Rajamani, "Chapter 2: Lateral vehicle dynamics," Springer Mechanical Engineering Series, pp. 15 - 46, 2012. 